\title{
Optimizing subject retention in a longitudinal birth cohort study: lessons learned from the Vancouver site of the CHILD Study
}

\author{
Linda Warner ${ }^{*}$, Mary Ann Mauro, Susan Menzies, Ghazal Assadian, Robby Mamonluk, Claire Lepine, Stuart E Turvey \\ From Canadian Society of Allergy and Clinical Immunology Annual Scientific Meeting 2013 \\ Toronto, Canada. 3-6 October 2013
}

\section{Background}

The Canadian Healthy Infant Longitudinal Development (CHILD) study is a multicentre birth cohort study following children for the first five years of life to determine how environmental and genetic variables impact early life health, particularly the development of asthma and allergies. Optimal subject retention is essential for scientific integrity, budget containment and ultimately, for continuity of data collection.

We completed an analysis of participants from the Vancouver general cohort after their first year in the study to address retention challenges related to urban mobility, time constraints, and stressful life situations while considering their socio-economic status (SES) in regards to family income and parents' education level.

\section{Methods}

Reasons for voluntary participant withdrawals were identified by questionnaires and by direct participant feedback. Anonymous surveys were administered to parents to evaluate clinical practice, and provide insight on changes that could be implemented.

\section{Results}

Out of 706 participants the CHILD study successfully retained $93 \%$ of participants with $3 \%$ excluded at birth due to exclusion criteria (e.g. premature birth, significant medical complication) and another $4 \%$ of the cohort voluntarily withdrawing. $11 \%$ of active participants were identified as participants at risk of withdrawing. Issues putting these participants at risk included: lack of time (25\%), difficulty with testing (23\%) and inconvenience of

\footnotetext{
* Correspondence: Iwarner@cw.bc.ca

Department of Pediatrics, University of British Columbia, Vancouver, BC, V6T 1Z4, Canada
} 